\title{
CD26 as a Promising Biomarker for Predicting Prognosis in Patients with Pancreatic Tumors
}

This article was published in the following Dove Press journal: OncoTargets and Therapy

\author{
Liang Yan ${ }^{1, *}$ \\ Xiuyun $\operatorname{Tian}^{1, *}$ \\ Chunxiang Ye (D) ${ }^{1,2}$ \\ Xiaoya Guan' \\ Bin Dong ${ }^{3}$ \\ Min Zhao ${ }^{4}$ \\ Jianhui Wu' \\ Chunyi Hao iD ${ }^{\prime}$ \\ 'Key Laboratory of Carcinogenesis and \\ Translational Research (Ministry of \\ Education/Beijing), Department of \\ Hepato-Pancreato-Biliary Surgery, Peking \\ University Cancer Hospital \& Institute, \\ Beijing, People's Republic of China; \\ ${ }^{2}$ Department of General Surgery, Beijing \\ Chao-Yang Hospital, Capital Medical \\ University, Beijing, People's Republic of \\ China; ${ }^{3}$ Key Laboratory of \\ Carcinogenesis and Translational \\ Research (Ministry of Education/Beijing), \\ Central Laboratory, Peking University \\ Cancer Hospital \& Institute, Beijing, \\ People's Republic of China; ${ }^{4}$ Key \\ Laboratory of Carcinogenesis and \\ Translational Research (Ministry of \\ Education/Beijing), Department of \\ Pathology, Peking University Cancer \\ Hospital \& Institute, Beijing, People's \\ Republic of China
}

*These authors contributed equally to this work

Correspondence: Chunyi Hao Key Laboratory of Carcinogenesis and Translational Research (Ministry of Education/Beijing), Department of Hepato-Pancreato-Biliary Surgery, Peking University Cancer Hospital \& Institute, No. 52 Fucheng Road, Haidian District, Beijing, People's Republic of China

Tel +86- I0-88|96|82

$\mathrm{Fax}+86-10-88196548$

Email haochunyi@bjmu.edu.cn
Purpose: Pancreatic cancer is associated with a high mortality rate owing to insufficient approaches for early diagnosis and the invasive biological behavior of the cancer. CD26 is a membrane-anchored protein involved in multiple physiological and pathological processes. Here, we investigated correlations between CD26 expression and clinicopathological features in patients with pancreatic tumors.

Methods: We collected 170 tumor tissue specimens and 138 paired paratumoral tissues from patients with pancreatic tumors and evaluated CD26 expression using immunohistochemistry.

Results: CD26 was expressed in $79.4 \%$ of pancreatic tumors, which was significantly $(P<$ $0.001)$ higher than that in paratumoral pancreatic tissues $(23.2 \%)$. High expression of CD26 was correlated with ABO blood type $(P=0.035)$, malignancy degree $(P=0.001)$, CA199 $(P=0.01)$, and CA242 $(P=0.027)$. In pancreatic malignancies, CD26 expression was observed in $80.7 \%(130 / 161)$ of cases. Lower CD26 expression was correlated with longer disease-free survival $(P=0.048)$ and overall survival $(P=0.024)$ and was an independent predictor of overall survival (hazard ratio $[\mathrm{HR}]$ : $1.713 ; P=0.042$ ). Similar results were observed in pancreatic ductal adenocarcinoma (PDAC) tissues, and CD26 expression level (HR: $2.117 ; P=0.008$ ) was an independent predictor of overall survival in patients with PDAC. CD26 expression was significantly increased in pancreatic tumors and gradually increased with increasing malignancy degree, suggesting that CD26 may be involved in the tumorigenic proliferation of pancreatic tumors.

Conclusion: Therefore, CD26 is a potential marker for early diagnosis and a promising therapeutic target in pancreatic tumors.

Keywords: biomarker, CD26, immunohistochemistry, pancreatic cancer, prognosis

\section{Introduction}

Pancreatic cancer is associated with a high mortality rate and was previously reported to be the fourth leading cause of cancer-related death in the United States of America. ${ }^{1}$ In recent years, important advances have been made in the diagnosis and treatment of pancreatic cancer. However, in 2015, according to Chinese cancer registration data, the estimated mortality rate of pancreatic cancer was still similar to the estimated incidence $\left(79,400\right.$ versus 90,100 cases). ${ }^{2}$ The high mortality may be related to the lack of symptoms during early-stage disease and the high potential for invasion and metastasis of pancreatic cancer cells. Therefore, exploration of biomarkers that may be involved in tumorigenesis and have potential applications in early diagnosis and as treatment targets are urgently required.

CD26, also known as dipeptidyl peptidase 4 (DPPIV), is a serine peptidase that belongs to the dipeptidyl peptidase family $9 \mathrm{~b} .^{3}$ This multifunctional protein has 
proline-specific dipeptidyl peptidase activity that is involved in diverse biological processes, such as $\mathrm{T}$ cell activation, and may cleave several chemokines under physiological conditions. ${ }^{4}$ The major substrates of CD26 are gastric inhibitory polypeptide and glucagon-like peptide-1; thus, CD26 inhibitors have been used to treat type II diabetes. ${ }^{5}$ Moreover, CD26 was recently found to be overexpressed in several types of cancers, including malignant mesothelioma, ${ }^{6}$ thyroid carcinoma, ${ }^{7}$ and urothelial carcinoma. ${ }^{8}$ Our previous work showed that CD26 overexpression promotes the growth and invasion of pancreatic cancer cells, whereas suppression of CD26 inhibits malignant behaviors. ${ }^{9}$ However, CD26 expression in specimens from patients with pancreatic tumors has not been extensively investigated.

Accordingly, in this study, we aimed to investigate CD26 expression in benign and malignant pancreatic tumors and to clarify the correlations between CD26 expression and clinicopathological features or prognosis in patients with pancreatic cancer. Our findings are expected to help elucidate the mechanisms of pancreatic cancer progression and to establish CD26 as a potential prognostic factor and therapeutic target.

\section{Patients and Methods}

\section{Patients and Samples}

In total, 170 patients with pancreatic masses who underwent surgical treatment at the Department of HepatoPancreato-Biliary Surgery of Peking University Cancer Hospital during 2011-2017 were included in this study. Among these 170 cases, 109 were cases of pancreatic ductal adenocarcinoma and its variants (PDACs), 28 were cases of solid-pseudopapillary neoplasms (SPTs), 18 were cases of neuroendocrine neoplasms (NENs), three were cases of serous neoplasms, two were cases of mucinous neoplasms (MCNs), three were cases of intraductal papillary mucinous neoplasms (IPMNs), three were cases of pancreatitis, one was a case of acinar cell neoplasm, and three were cases of secondary pancreatic metastasis. Patients who received adjuvant chemotherapy or radiotherapy were excluded. During the experimental process, 32 paratumoral tissues were lost during processing or were too small for evaluation and were thus unable to be assessed. Overall, there were 170 pancreatic tumor specimens and 138 paired paratumoral tissues included in subsequent analyses. The average age of the 170 patients with pancreatic tumors was $55.9 \pm 15.6$ years old. The detailed baseline features of all patients are described in Table S1. Tumor specimens and paired paratumoral tissues were immediately fixed in formalin within $30 \mathrm{~min}$ after resection and then embedded with paraffin for long-term conservation. Specimens were cut into $4-\mu \mathrm{m}$-thick slices for staining.

\section{Immunohistochemical Analysis}

Formalin-fixed, paraffin-embedded specimens were baked at $60^{\circ} \mathrm{C}$ for $2 \mathrm{~h}$, dewaxed in xylene, and rehydrated through graded concentrations of alcohol. Antigen retrieval was performed with ethylenediaminetetraacetic acid (pH $\quad 8.0$; Beijing Zhongshan Golden Bridge Biotechnology Co., Ltd., China) for $2.5 \mathrm{~min}$ in a pressure cooker. After natural cooling to room temperature, the slices were washed with phosphate-buffered saline (PBS, pH 7.0; Beijing Zhongshan Golden Bridge Biotechnology Co., Ltd.) three times. Slices were then soaked in $3 \%$ hydrogen peroxide for $15 \mathrm{~min}$ to eliminate endogenous peroxidase activity. After washing in PBS three times, the sections were blocked with goat serum (Beijing Zhongshan Golden Bridge Biotechnology Co., Ltd.) at $37^{\circ} \mathrm{C}$ for $1 \mathrm{~h}$, followed by incubation with primary rabbit polyclonal anti-DPPIV antibodies $(1: 1200$; cat. no. NB100-59021; Novus Biologicals, USA) overnight at $4^{\circ}$ C. Unconjugated primary antibody was then removed by washing in PBS, and ready-to-use EnVision reagent (EnVision Detection System Peroxidase/DAB, Rabbit/ Mouse; Dako, Denmark) was used to bind the primary antibody. A 3,3'-diaminobenzidine tetrahydrochloride kit (EnVision Detection System Peroxidase/DAB, Rabbit/ Mouse; Dako) was used to develop substrates. After counterstaining with hematoxylin, the sections were dehydrated and transparentized with graded alcohol and xylene. Positivity for anti-CD26 antibody staining was determined in epithelial ovarian cancer and colorectal tumors (Figure S1).

\section{Evaluation of Staining}

Two independent pathologists who were blinded to the clinical data of the patients examined and scored CD26 expression under a microscope using the immunoreactivity score (IRS) system. The IRS was the product of the percentage of positive cells and staining intensity. IRSs of $0-1,2-4,5-8$, and 9-12 were evaluated as "-”, “+”, “+ + ", and "+++", respectively. In subsequent analyses, "-" and "+" were considered low CD26 low expression, and "++" and "+++" were considered high CD26 expression. 


\section{Statistical Analysis}

SPSS Statistics (version 22) was used to perform statistical analysis. Two-tailed $\chi^{2}$ or Fisher tests were used to compare the expression of CD26 between different groups. In order to assess the correlations between CD26 expression and clinicopathological parameters, we used $\chi^{2}$ tests and Spearman's rank correlation analysis. Overall survival (OS) rates and disease-free survival (DFS) rates were analyzed by KaplanMeier survival tests, and $p$ values were calculated using Log rank tests to evaluate the correlations between prognosis and CD26 expression. Univariate and multivariate survival analyses were performed with Cox proportional hazard regression model to identify independent parameters affecting overall survival. Results with $p$ values of less than 0.05 were considered significant.

\section{Results}

\section{CD26 Expression Was Markedly Higher in Pancreatic Tumor Tissues Than in Paratumoral Tissues}

The 170 cases of pancreatic tumors were grouped into nine pathological types according to the World Health Organization Classification of Tumors of the Digestive System. Of the 170 cases, PDACs accounted for $64.1 \%$; SPTs accounted for $16.5 \%$; NENs accounted for $10.6 \%$; serous neoplasms, IPMNs, pancreatitis, and secondary pancreatic metastases each accounted for $1.8 \%$; MCNs accounted for $1.2 \%$; and acinar cell neoplasms accounted for only $0.6 \%$ (Table S1).

CD26 expression was assessed in these cases using immunohistochemistry. Of the 170 tumor specimens, 135 were CD26 positive, whereas only 32 of the 138 paratumoral tissues were positive. CD26 expression was markedly higher in tumor tissues than in paratumoral tissues (79.4\% versus $23.2 \%$, respectively; $P<0.001$; Table 1 ).

\section{CD26 Expression Was Significantly Higher in PDACs, NENs, and SPTs}

The positive rates of CD26 expression in tumor and noncancerous tissues were $82.6 \%$ versus $31.4 \%$, respectively, in PDACs (90/109 versus 27/86, respectively; $P<0.001)$; $83.3 \%$ versus $15.4 \%$, respectively, in NENs $(15 / 18$ versus $2 / 13$, respectively; $P<0.001$ ); and $78.6 \%$ versus $4.0 \%$, respectively, in SPTs (22/28 versus $1 / 25$, respectively; $P<$ $0.001)$. No statistical significance was found in other types of pancreatic tumors owing to the small sample size. All
Table I CD26 Expression of 170 Pancreatic Tumor Patients

\begin{tabular}{|c|c|c|c|c|}
\hline & Total & $\begin{array}{l}\text { CD26 } \\
\text { Positive } \\
\text { [n (\%)] }\end{array}$ & $\begin{array}{l}\text { CD26 } \\
\text { Negative } \\
{[n(\%)]}\end{array}$ & $P$ value \\
\hline Total & 170 & & & \\
\hline $\mathrm{T}^{\mathrm{a}}$ & 170 & $135(79.4)$ & $35(20.6)$ & $P<0.001$ \\
\hline$N^{b}$ & 138 & $32(23.2)$ & $106(76.8)$ & \\
\hline PDAC & 109 & & & \\
\hline $\mathrm{T}$ & 109 & $90(82.6)$ & $19(17.4)$ & $P<0.001$ \\
\hline $\mathrm{N}$ & 86 & $27(31.4)$ & $59(68.6)$ & \\
\hline SPTs & 28 & & & \\
\hline $\mathrm{T}$ & 28 & $22(78.6)$ & $6(21.4)$ & $P<0.001$ \\
\hline $\mathrm{N}$ & 25 & $\mathrm{I}(4.0)$ & $24(96.0)$ & \\
\hline NENs & 18 & & & \\
\hline $\mathrm{T}$ & 18 & I5(83.3) & $3(16.7)$ & $P<0.00$ I \\
\hline $\mathrm{N}$ & 13 & $2(15.4)$ & II(84.6) & \\
\hline $\begin{array}{l}\text { Mucinous cystic } \\
\text { neoplasms }\end{array}$ & 2 & & & \\
\hline $\mathrm{T}$ & 2 & $I(50.0)$ & $I(50.0)$ & \\
\hline$N$ & 2 & $0(0)$ & $2(100.0)$ & \\
\hline $\begin{array}{l}\text { Serous } \\
\text { neoplasms }\end{array}$ & 3 & & & \\
\hline $\mathrm{T}$ & 3 & $3(100.0)$ & $0(0)$ & \\
\hline $\mathrm{N}$ & 3 & $0(0)$ & $3(100.0)$ & \\
\hline IPMNs & 3 & & & \\
\hline $\mathrm{T}$ & 3 & $\mathrm{I}(33.3)$ & $2(66.7)$ & \\
\hline $\mathrm{N}$ & 3 & $I(33.3)$ & $2(66.7)$ & \\
\hline Pancreatitis & 3 & & & \\
\hline $\mathrm{T}$ & 3 & $\mathrm{I}(33.3)$ & $2(66.7)$ & \\
\hline $\mathrm{N}$ & 2 & $I(50.0)$ & $I(50.0)$ & \\
\hline $\begin{array}{l}\text { Acinar cell } \\
\text { neoplasms }\end{array}$ & I & & & \\
\hline $\mathrm{T}$ & I & $0(0)$ & $I(100.0)$ & \\
\hline $\mathrm{N}$ & I & $0(0)$ & $I(100.0)$ & \\
\hline $\begin{array}{l}\text { Secondary } \\
\text { pancreatic } \\
\text { metastasis }\end{array}$ & 3 & & & \\
\hline $\mathrm{T}$ & 3 & $2(66.7)$ & $I(33.3)$ & \\
\hline $\mathrm{N}$ & 3 & $0(0)$ & $3(100.0)$ & \\
\hline
\end{tabular}

Notes: " pancreatic tissues"; The bold P value indicated significant difference.

data are shown in Table 1. Typical cytoplasmic staining of CD26 in various types of tumors is shown in Figure 1.

Pancreatic Tumors with Different Degrees of Malignancy Had Different CD26 Expression

According to the degree of malignancy, we classified the 170 cases of pancreatic tumors into three categories, ie, 

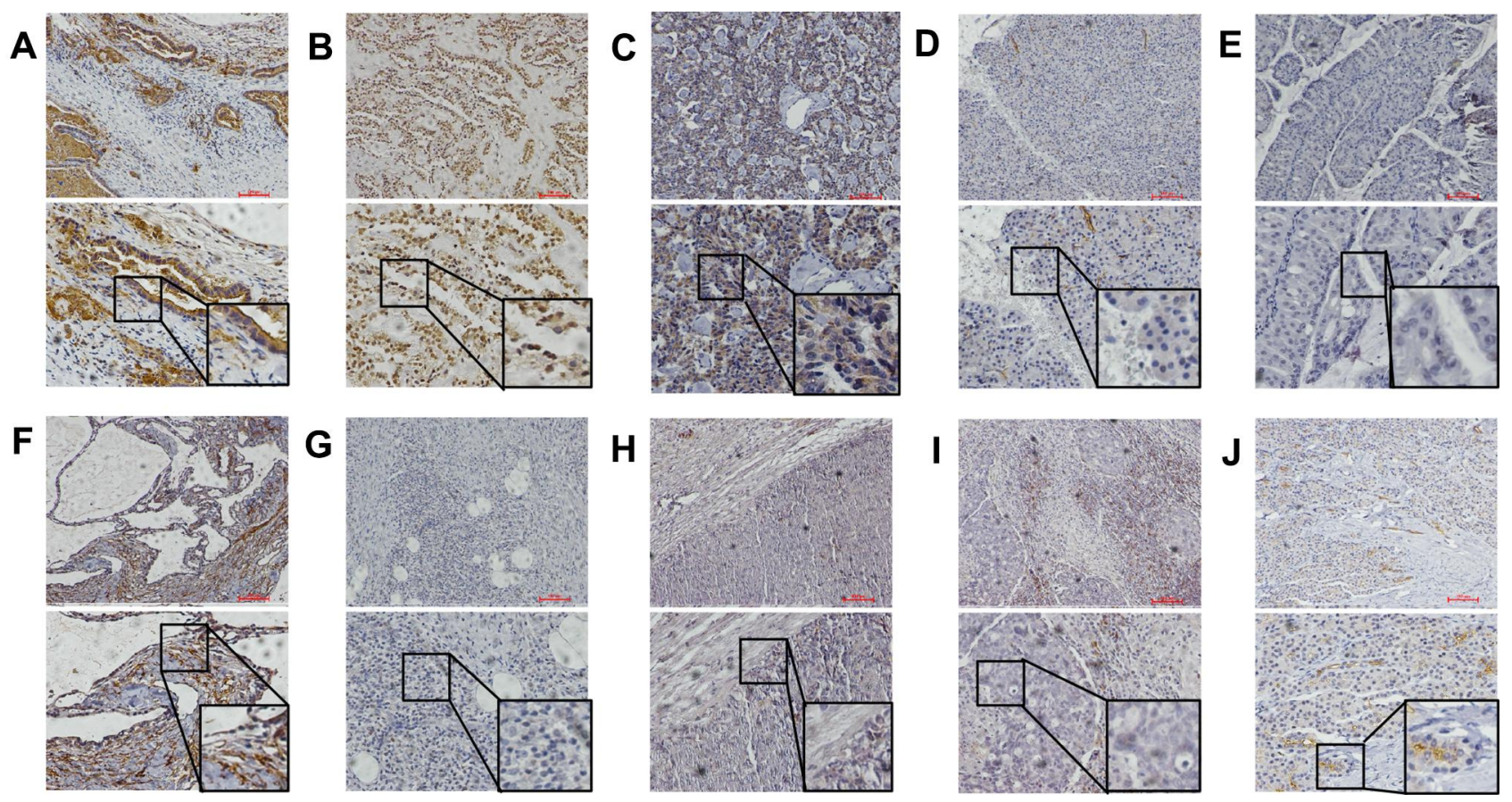

Figure I Typical cytoplasmic staining of CD26 in (A) PDAC; (B) SPT; (C) NEN; (D) paratumoral pancreatic tissue; (E) IPMN; (F) serous neoplasms; (G) pancreatitis; (H) acinar cell neoplasm; (I) secondary pancreatic metastasis from oviduct serous adenocarcinoma and $(\mathbf{J}) \mathrm{MCN}$. The upper ones of each figure were $\times 100$, and the lower ones were $\times 200$.

benign pancreatic tumors, low-grade malignant pancreatic tumors, and high-grade malignant pancreatic tumors. As shown in Figure 2, nine patients were histologically assessed as having benign tumors, among which the positive rate of CD26 expression was 55.6\% (5/9). Patients with SPTs were assessed as having low-grade malignant tumors, among which 22 cases were CD26 positive (78.6\%). The remaining pancreatic tumors were highgrade malignant tumors, exhibiting a positive rate of CD26 expression of $81.2 \%$ (108/133). Although there were no significant differences among benign, low-grade, and high-grade malignant pancreatic tumors $(P=0.239)$, high-grade malignant pancreatic tumors tend to have higher CD26 expression.

\section{Correlations Between CD26 Expression Levels and Clinicopathological Features in Patients with Pancreatic Tumors}

Next, we assessed the correlations between CD26 expression levels and clinical features in patients with pancreatic tumors. As shown in Tables 2 and 3, among 170 cases of pancreatic tumors, high CD26 expression was associated with ABO blood type $(P=0.035)$, tumor malignancy $(P=$ $0.001)$, CA199 $(P=0.010)$, and CA242 $(P=0.027)$. We did not find associations of high CD26 expression with sex
( $P=0.463)$, age $(P=0.119)$, smoking $(P=0.317)$, drinking $(P=0.852)$, abnormal blood glucose $(P=0.787)$, tumor size $(P=0.503)$, or tumor site $(P=0.175)$ for all patients.

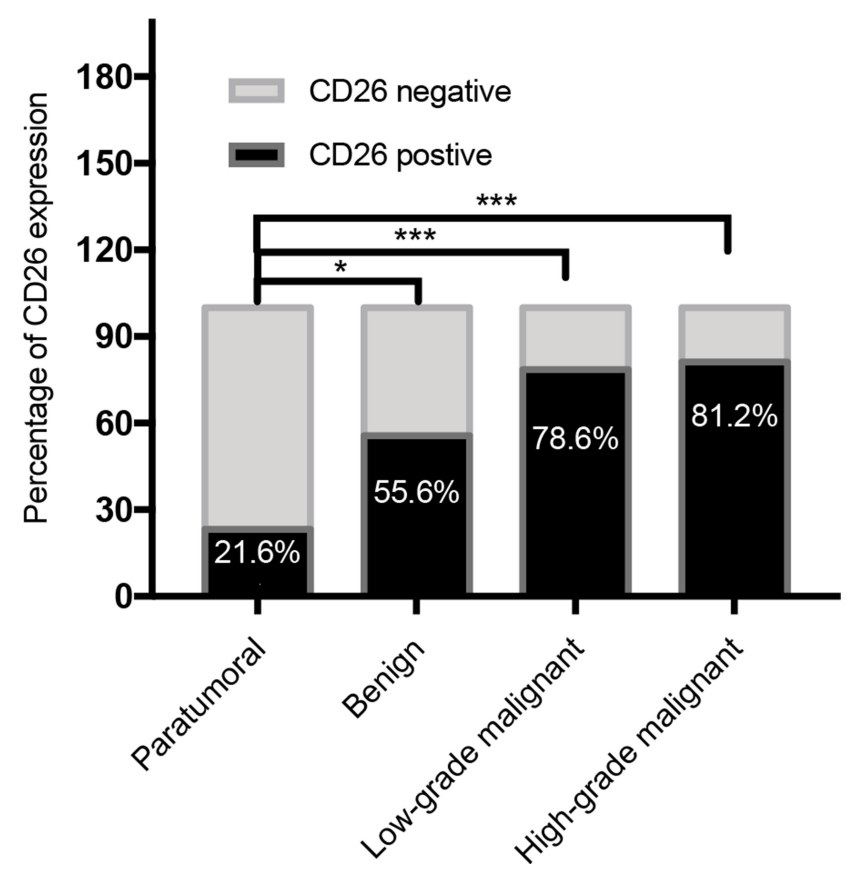

Figure 2 CD26 expression in paratumoral tissues, benign pancreatic tumors, lowgrade malignant pancreatic tumors and high-grade malignant pancreatic tumors. * represented $\mathrm{P}<0.05$, *** represented $\mathrm{P}<0.00 \mathrm{I}$. 
Table 2 Correlation Between CD26 Expression Level and Clinicopathological Features (Enumeration Data) in 170 Pancreatic Tumor Patients

\begin{tabular}{|c|c|c|c|}
\hline Characteristics & $\begin{array}{l}\text { CD26 High } \\
\text { Expression } \\
\text { [n (\%)] }\end{array}$ & $\begin{array}{l}\text { CD26 Low } \\
\text { Expression } \\
\text { [n (\%)] }\end{array}$ & $P$ value \\
\hline \multicolumn{4}{|l|}{ Gender } \\
\hline Male & $50(56.2)$ & $39(43.8)$ & 0.463 \\
\hline Female & $50(61.7)$ & 31 (38.3) & \\
\hline \multicolumn{4}{|l|}{ Smoking ${ }^{\mathrm{a}}$} \\
\hline Deny & $73(57.0)$ & $55(43.0)$ & 0.317 \\
\hline Present & $27(65.9)$ & $14(34.1)$ & \\
\hline \multicolumn{4}{|l|}{ Drinking $^{\mathrm{a}}$} \\
\hline Deny & $80(58.8)$ & $56(4 \mid .2)$ & 0.852 \\
\hline Present & $0(0.0)$ & $13(100.0)$ & \\
\hline \multicolumn{4}{|l|}{$\begin{array}{l}\text { Abnormal blood } \\
\text { glucose }^{\mathrm{a}}\end{array}$} \\
\hline Absent & $75(58.6)$ & $53(41.4)$ & 0.787 \\
\hline Present & $25(61.0)$ & $16(39.0)$ & \\
\hline \multicolumn{4}{|l|}{ Blood type $^{a}$} \\
\hline A type & $39(60.9)$ & $25(39.1)$ & 0.035 \\
\hline B type & $21(55.3)$ & 17 (44.7) & \\
\hline$A B$ type & $16(84.2)$ & $3(15.8)$ & \\
\hline O type & $21(45.7)$ & $25(54.3)$ & \\
\hline \multicolumn{4}{|l|}{ Tumor site } \\
\hline Head & $46(56.1)$ & $36(43.9)$ & 0.175 \\
\hline Body and tail & $51(60.0)$ & $34(40.0)$ & \\
\hline Whole pancreas & $3(100.0)$ & $0(0.0)$ & \\
\hline \multicolumn{4}{|l|}{ Malignancy } \\
\hline Benign & $3(33.3)$ & $6(66.7)$ & 0.001 \\
\hline Low-grade malignant & $9(32.1)$ & $19(67.9)$ & \\
\hline High-grade malignant & $88(66.2)$ & $45(33.8)$ & \\
\hline
\end{tabular}

Notes: aClinicopathological data of several patients were incomplete, yet do not affect the overall results; The bold $\mathrm{P}$ value indicated significant difference.

\section{High CD26 Expression was Correlated with Pathological Type and Serum Tumor Markers in Patients with Malignant Pancreatic Tumors}

Further analyses were conducted among the 161 patients with malignant pancreatic tumors. The results showed that high CD26 expression was associated with pathological type $(P=0.001)$, CA199 $(P=0.013)$, and CA242 $(P=$ 0.025), but not with sex $(P=0.174)$, age $(P=0.056)$, smoking $(P=0.132)$, drinking $(P=0.808)$, abnormal blood glucose $(P=0.547)$, blood type $(P=0.052)$, tumor size $(P=0.490)$, tumor site $(P=0.213)$, blood vessel invasion $(P=0.600)$, tumor invasion $(P=0.317)$,
Table 3 Correlation Between CD26 Expression Level and Clinicopathological Features (Measurement Data) in 170 Pancreatic Tumor Patients

\begin{tabular}{|l|l|l|}
\hline $\begin{array}{l}\text { Clinicopathological } \\
\text { Features }\end{array}$ & $\begin{array}{l}\text { Correlation of Coefficient } \\
\text { with CD26 Expression }\end{array}$ & $\boldsymbol{P}$ value \\
\hline Age & 0.120 & 0.119 \\
Size & -0.052 & 0.503 \\
CEA & 0.087 & 0.291 \\
CA199 & 0.210 & $\mathbf{0 . 0 1 0}$ \\
CA72.4 & 0.082 & 0.324 \\
CA242 & 0.197 & $\mathbf{0 . 0 2 7}$ \\
Disease-free survival & -0.348 & $<\mathbf{0 . 0 0 1}$ \\
time (months) & & $<0.001$ \\
Overall survival time & -0.310 & \\
(months) & \multicolumn{2}{|}{} \\
\hline
\end{tabular}

Note: The bold $\mathrm{P}$ value indicated significant difference.

lymph node metastasis $(P=0.444)$, distant metastasis $(P=$ $0.246)$, tumor stage $(P=0.451)$, carcinoembryonic antigen $(P=0.174)$, or CA72.4 $(P=0.351$; Tables S2 and $\underline{\mathrm{S} 3})$.

Next, subgroup analyses were performed in PDACs, NENs, and SPTs. High CD26 expression was found to be associated with $\mathrm{ABO}$ blood type $(P=0.033)$ in patients with PDAC. No other significant relationships were found in these patients (data not shown).

\section{Higher CD26 Expression was Correlated with Poorer Overall Survival in Patients with Malignant Pancreatic Tumors}

Among malignant pancreatic tumors, Kaplan-Meier survival analysis and log rank tests showed that higher CD26 expression was associated with poorer DFS $(P=0.048)$ and poorer OS $(P=0.024)$, as shown in Figure $3 \mathrm{~A}$ and $\mathrm{B}$. The median DFS times were $47.00 \pm 5.02$ months in the high CD26 expression group and $47.80 \pm 4.04$ months in the low CD26 expression group. The median OS times were $31.43 \pm 4.00$ months in the high CD26 expression group and $38.43 \pm 3.71$ months in the low CD26 expression group.

Various clinicopathological features that may affect DFS and OS in patients with malignant pancreatic tumors were evaluated by univariate survival analysis. Tumor pathological type (hazard ratio [HR]: 4.259; 95\% confidence interval [CI]: 1.015-17.879; $P=0.048)$, distant metastasis (HR: 194.821; 95\% CI: 42.064-902.316; $P<$ 0.001 ), and tumor stage (HR: $10.842 ; 95 \%$ CI: 5.360 21.929; $P<0.001)$ were confirmed to be prognostic factors of DFS. These prognostic factors and CD26 

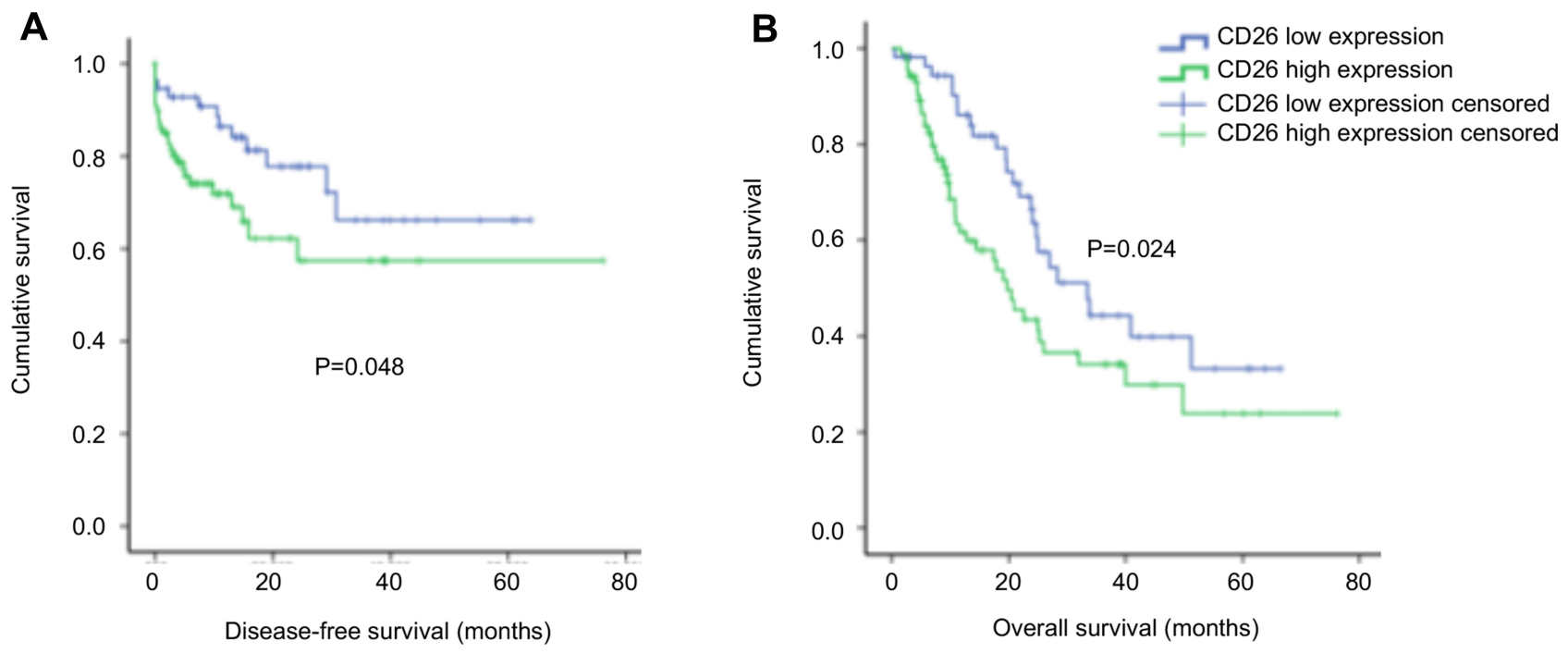

Figure 3 Higher CD26 expression was associated with poorer DFS and OS in malignant pancreatic tumor patients. According to CD26 expression level, all the malignant pancreatic tumor patients were divided into high expression group and low expression group. Kaplan-Meier survival analysis and Log rank test showed that patients with lower CD26 expression had a better $(\mathbf{A})$ disease-free survival and $(\mathbf{B})$ overall survival than those with higher $C D 26$ expression $(P=0.048$ and $P=0.024)$.

expression were included in the Cox multivariate model. Only tumor stage (HR: 10.558; 95\% CI: 5.156-21.622; $P<0.001)$ was shown to be an independent prognostic factor for DFS (Table S4).

Univariate survival analysis suggested that age (HR: 1.045; 95\% CI: 1.025-1.064; $P<0.001$ ), lymph node metastasis (HR: 1.862; 95\% CI: 1.126-3.081; $P=$ 0.016), tumor stage (HR: $2.031 ; 95 \%$ CI: $1.171-3.524$; $P=0.012$ ), and high CD26 expression (HR: $1.774 ; 95 \%$ CI: $1.071-2.939 ; P=0.026$ ) were prognostic factors of OS. Cox multivariate survival analyses showed that age
(HR: 1.048 ; 95\% CI: $1.025-1.071 ; P<0.001)$, tumor stage (HR: 2.364; 95\% CI: $1.337-4.179 ; P=0.003$ ), and high CD26 expression (HR: 1.713; 95\% CI: 1.019-2.880; $P=$ 0.042 ) were independent prognostic factors for OS. Detailed information is shown in Table 4.

Further subgroup analyses showed that patients with PDAC with lower CD26 expression had favorable OS compared with those with higher CD26 expression $(P=0.023)$. The median OS times were $29.38 \pm 3.93$ months for patients with low CD26 expression and $17.93 \pm 1.96$ months for patients with high CD26 expression (Figure S2). No other

Table 4 Cox Proportional Hazard Regression Model Analysis of Overall Survival in I6I Malignant Pancreatic Cancer Patients

\begin{tabular}{|c|c|c|c|c|c|c|}
\hline \multirow[t]{2}{*}{ Characteristics } & \multicolumn{3}{|c|}{ Univariate Analysis } & \multicolumn{3}{|c|}{ Multivariate Analysis } \\
\hline & $P$ value & Hazard Ratio & 95\% Confidence Interval & $P$ value & Hazard Ratio & 95\% Confidence Interva \\
\hline Gender & 0.377 & 1.245 & $0766-2.025$ & & & \\
\hline Age (years) & $<0.001$ & 1.045 & $1.025-1.064$ & $<0.001$ & 1.048 & $1.025-1.07 \mid$ \\
\hline Smoking & 0.080 & 1.602 & $0.944-2.719$ & & & \\
\hline Drinking & 0.783 & 0.915 & $0.488-1.717$ & & & \\
\hline Abnormal blood glucose & 0.090 & 1.594 & $0.930-2.732$ & & & \\
\hline Blood type & 0.489 & 1.221 & $0.694-2.148$ & & & \\
\hline Size & 0.965 & 1.002 & $0.909-1.105$ & & & \\
\hline Blood vessel invasion & 0.342 & 1.272 & $0.774-2.089$ & & & \\
\hline Tumor invasion & 0.056 & 1.644 & $0.987-2.740$ & & & \\
\hline Lymph node metastasis & 0.016 & 1.862 & $1.126-3.081$ & & & \\
\hline Metastasis & 0.124 & 1.754 & $0.857-3.591$ & & & \\
\hline Tumor stage & 0.012 & 2.031 & $1.17 \mid-3.524$ & 0.003 & 2.364 & $1.337-4.179$ \\
\hline CD26 level & 0.026 & 1.774 & $1.07 \mathrm{I}-2.939$ & 0.042 & 1.713 & $1.019-2.880$ \\
\hline
\end{tabular}

Note: The bold $P$ value indicated significant difference. 
significant results were found in NENs or SPTs owing to the small sample size and lower malignancy of the tumors; detailed information is shown in Figure S3.

Finally, we performed univariate and multivariate survival analyses of DFS and OS in PDACs. Similar to the results for all pancreatic malignancies (Tables S5 and S6), tumor stage (HR: 8.689; 95\% CI: 3.986-18.943; $P<$ 0.001) was an independent factor affecting DFS. Tumor stage (HR: 3.398; 95\% CI: 1.879-6.145; $P<0.001$ ) and higher CD26 expression (HR: 2.117; 95\% CI: 1.214 3.691; $P=0.008$ ) were independent prognostic factors in patients with PDAC.

\section{Discussion}

CD26 was first characterized as a type II transmembrane glycoprotein of approximately $110 \mathrm{kDa}$ and was showed to have peptidase activity that could cleave oligopeptides with an L-proline or L-alanine at the penultimate position into dipeptides from the N-terminal.,10 CD26 is a multifunctional serine proteinase involved in various physiological processes, including immune modulation and signal transduction, and pathological processes, such as inflammation, autoimmune diseases, and tumor invasion. ${ }^{10}$ Furthermore, CD26/DPPIV cleaves multiple substrates with broad functions.

Importantly, CD26 plays various roles in different tumors. ${ }^{10,11}$ In this study, CD26 expression was found to be markedly higher in tumor tissues than in paratumoral tissues. Detailed analyses of the pathological subtypes in patients with pancreatic tumors revealed that positive rates of CD26 expression in patients with PDACs, SPTs, NENs, and benign pancreatic tumors were $82.6 \%, 78.6 \%, 83.3 \%$, and $55.7 \%$, respectively. Busek et al have evaluated the enzymatic activity of CD26 in pancreatic tumor tissues and circulation of PDAC patients and found that $95 \%$ of the tumors expressed CD26. ${ }^{12}$ Furthermore, Arias-Pinilla et al have shown that $61 \%$ of the 34 tissue samples expressed CD26. ${ }^{13}$ These two studies are consistent with our results. Here, we assessed CD26 expression using a larger sample size and analyzed in detail its correlation with various clinicopathological features. We further determined its expression in NENs and SPTs. Our results indicated that CD26 was aberrantly expressed in pancreatic tumors and may be involved in pancreatic tumorigenesis as an oncogene, which corroborates the findings of our previous study. ${ }^{9}$ Similarly to our results, CD26 was overexpressed in patients with thyroid cancer, ${ }^{7,14}$ malignant mesothelioma, ${ }^{15}$ ovarian carcinoma, ${ }^{16}$ and HCC. $^{17}$
Particularly, in patients with HCC, CD26 overexpression was correlated with the proliferation marker ki-67 in tumor cells, thereby indicating that CD26 expression may be involved in tumorigenic proliferation. ${ }^{17}$ However, in melanoma, decreased CD26 expression was found in tumor tissues, and subsequently, its role in tumor suppression has been suggested. ${ }^{18}$ The diverse roles of CD26 may be attributed to the various ligands it interacts with in different kinds of cancers and its specific location in tumor cells. In malignant mesothelioma, CD26 was mainly overexpressed in the cytoplasm, whereas surface expression was lacking. In pancreatic tumors, we similarly found that CD26 was expressed in the cytoplasm, the cytoplasmic region of CD26 was associated with tumor growth through inhibiting the somatostatin receptor 4 in malignant mesothelioma. ${ }^{19}$ Thus, the mechanism of CD26 in pancreatic tumors needs to be elucidated.

The diagnostic value of CD26 in distinguishing between benign, low-grade malignant, and high-grade malignant pancreatic tumors was assessed, and we found that CD26 was expressed in $55.6 \%$ of benign, $78.6 \%$ of low-grade malignant, and $81.2 \%$ of high-grade malignant pancreatic tumors. Although these differences were not statistically significant, the findings suggested that CD26 expression was relatively higher in tumors with high-grade malignancy, which may be due to the high proliferation activity in high-grade malignancies. A similar trend was also observed in thyroid tumors, ovarian tumors, and malignant mesotheliomas. ${ }^{15,16,20,21}$ In pancreatic tumors, the diagnostic value of CD26 is understudied. Differences in CD26 expression among paratumoral tissues, precancerous lesions (eg, panINs), and pancreatic cancers should be investigated to confirm the diagnostic value of CD26.

In pancreatic malignancies, positive expression of CD26 was associated with tumor invasion (T1, T2, T3, T4: $56.0 \%, 87.8 \%, 84.1 \%$, and $75.0 \%$, respectively; $P=$ 0.013; detailed data not shown). This result was similar to the results of previous studies in thyroid cancer ${ }^{7}$ and urothelial carcinoma. ${ }^{8}$ Our results showed that patients with distant metastases had lower CD26 expression; therefore, we further performed CD26 IHC staining in cancer tissues, paired noncancerous tissues, and liver metastases from four patients with pancreatic cancer (Figure S4). Our results showed that CD26 expression was generally lower in metastatic specimens than primary tumor tissues and was not stronger than that in paratumoral hepatic tissues. Based on the various functions of CD26, CD26 may be involved in tumor invasion and metastasis by cooperating 
with other proteins or its substrates. For example, CD26, together with fibroblast activation protein, is involved in the degradation of the extracellular matrix, which has roles in tumor invasion. ${ }^{23}$ Additionally, overexpression of CD26 increases adhesion potential between ovarian cancer cells and mesothelium cells mediated by fibronectin. ${ }^{24}$ Further investigations are still needed to elucidate the different roles of $\mathrm{CD} 26$ in pancreatic cancer. Interestingly, we found that CD26 expression was related to ABO blood type. Consistent with this finding, a previous study has shown that the ABO blood type correlates with the risk of pancreatic cancer and diabetes. ${ }^{22}$ However, further studies are needed to clarify the potential correlations between ABO blood type, CD26 expression, and pancreatic diseases.

Unlike in other cancers, ${ }^{8,25-27}$ in pancreatic malignancies, the expression status of CD26 was not associated with DFS or OS, which may be attributed to the high positive rate. However, when patients were classified into CD26 high-/low-expression groups, higher CD26 expression was correlated with poorer DFS and OS and was an independent predictive factor of OS. In addition, we also found that the level of CD26 expression was correlated with CA199 and CA242, suggesting that CD26 may have applications as an early marker of pancreatic tumorigenesis and that CD26 expression level may be a predictor of prognosis. Furthermore, our previous study showed that CD26 expression was higher in serum samples from patients with pancreatic cancer than in that from healthy donors, and postoperation serum CD26 levels decreased compared with the corresponding level before the operation. ${ }^{28}$ Therefore, future studies are needed to determine the prognostic significance of combined serum CD26 levels and CD26 immunostaining to monitor the progression of pancreatic cancer. In our analysis of 109 patients with PDAC, we did not find any correlations between CD26 expression and clinicopathological features. Univariate and multivariate analyses showed that higher CD26 expression and tumor stage were independent factors of OS, but not DFS. In patients with NEN and SPT, CD26 expression level was not associated with OS or DFS as these two types of pancreatic tumors were relatively less malignant. Moreover, although we observed the separation of DFS curves, log-rank analysis showed that there was no significant difference, potentially because of the relatively small sample size. Accordingly, further studies with larger sample sizes and longer followup are needed to validate our findings.

\section{Conclusions}

In conclusion, we found that pancreatic tumorigenesis and progression were closely associated with CD26 overexpression. Additionally, CD26 expression was higher in tumors with high-grade malignancy. Thus, CD26 may have potential applications in the early and differential diagnosis and treatment of pancreatic tumors.

\section{Ethics Approval and Informed Consent}

All patients provided written informed consent for participation in the study. The study protocol was in accordance with the Declaration of Helsinki, and the research ethics committee of Peking University Cancer Hospital approved this study (approval no. 2016KT41).

\section{Consent for Publication}

The manuscript is approved by all authors for publication.

\section{Acknowledgment}

We would like to thank Editage (www.editage.cn) for English language editing.

\section{Funding}

This study was supported by Beijing Municipal Administration of Hospitals Clinical Medicine Development of Special Funding Support (grant no. XMLX201708), Capital Health Research and Development of Special Funds (approval No.: 2020-1-1021), Beijing Municipal Administration of Hospital's Ascent Plan (grant no. DFL20181104), the National Natural Science Funding (grant no. 31770836 and 81673500), Beijing Municipal Administration of Hospitals' Youth Programme (approval No. QML20181104), Interdisciplinary Medicine Seed Fund of Peking University and the Fundamental Research Funds for the Central Universities [approval No.: BMU2020MX015], and Science Foundation of Peking University Cancer Hospital 2020-13 and 2020-14.

\section{Disclosure}

The authors have no competing interests with regard to this study.

\section{References}

1. Hidalgo M. Pancreatic cancer. $N$ Engl J Med. 2010;362 (17):1605-1617. doi:10.1056/NEJMra0901557

2. Chen W, Zheng R, Baade PD, et al. Cancer statistics in China, 2015. CA Cancer J Clin. 2016;66(2):115-132. doi:10.3322/caac.21338 
3. Wagner L, Klemann C, Stephan M, von Horsten S. Unravelling the immunological roles of dipeptidyl peptidase 4 (DPP4) activity and/or structure homologue (DASH) proteins. Clin Exp Immunol. 2016;184 (3):265-283. doi:10.1111/cei.12757

4. Klemann C, Wagner L, Stephan M, von Horsten S. Cut to the chase: a review of CD26/dipeptidyl peptidase-4's (DPP4) entanglement in the immune system. Clin Exp Immunol. 2016;185(1):1-21. doi:10.11 11/cei.12781

5. Tseng CM, Liao WC, Chang CY, et al. Incretin-based pharmacotherapy and risk of adverse pancreatic events in the ethnic Chinese with diabetes mellitus: a population-based study in Taiwan. Pancreatology. 2017;17(1):76-82. doi:10.1016/j.pan.2016.10.003

6. Aoe K, Amatya VJ, Fujimoto N, et al. CD26 overexpression is associated with prolonged survival and enhanced chemosensitivity in malignant pleural mesothelioma. Clin Cancer Res. 2012;18 (5):1447-1456.

7. Lee JJ, Wang TY, Liu CL, et al. Dipeptidyl Peptidase IV as a prognostic marker and therapeutic target in papillary thyroid carcinoma. J Clin Endocrinol Metab. 2017;102(8):2930-2940. doi:10.1210/jc.2017-00346

8. Liang PI, Yeh BW, Li WM, et al. DPP4/CD26 overexpression in urothelial carcinoma confers an independent prognostic impact and correlates with intrinsic biological aggressiveness. Oncotarget. 2017;8(2):2995-3008. doi:10.18632/oncotarget.13820

9. Ye C, Tian X, Yue G, et al. Suppression of CD26 inhibits growth and metastasis of pancreatic cancer. Tumour Biol. 2016;37(12): 15677-15686. doi:10.1007/s13277-016-5315-4

10. Enz N, Vliegen G, De Meester I, Jungraithmayr W. CD26/DPP4 a potential biomarker and target for cancer therapy. Pharmacol Ther 2019;198:135-159. doi:10.1016/j.pharmthera.2019.02.015

11. Beckenkamp A, Davies S, Willig JB, Buffon A. DPPIV/CD26: a tumor suppressor or a marker of malignancy? Tumour Biol. 2016;37(6):7059-7073. doi:10.1007/s13277-016-5005-2

12. Busek $P$, Vanickova $Z$, Hrabal $P$, et al. Increased tissue and circulating levels of dipeptidyl peptidase-IV enzymatic activity in patients with pancreatic ductal adenocarcinoma. Pancreatology. 2016;16(5): 829-838.

13. Arias-Pinilla GA, Dalgleish AG, Mudan S, Bagwan I, Walker AJ, Modjtahedi H. Development and application of two novel monoclonal antibodies against overexpressed CD26 and integrin $\alpha 3$ in human pancreatic cancer. Sci Rep. 2020;10(1):1-13. doi:10.1038/s41598019-57287-w

14. Kotani T, Aratake Y, Ogata Y, et al. Expression of dipeptidyl aminopeptidase IV activity in thyroid carcinoma. Cancer Lett. 1991;57 (3):203-208. doi:10.1016/0304-3835(91)90158-E

15. Inamoto $\mathrm{T}$, Yamada $\mathrm{T}$, Ohnuma $\mathrm{K}$, et al. Humanized anti-CD26 monoclonal antibody as a treatment for malignant mesothelioma tumors. Clin Cancer Res. 2007;13(14):4191-4200. doi:10.1158/ 1078-0432.CCR-07-0110
16. Zhang M, Xu L, Wang X, Sun B, Ding J. Expression levels of seprase/FAP $\alpha$ and DPPIV/CD26 in epithelial ovarian carcinoma. Oncol Lett. 2015;10(1):34-42. doi:10.3892/ol.2015.3151

17. Nishina S, Yamauchi A, Kawaguchi T, et al. Dipeptidyl Peptidase 4 inhibitors reduce hepatocellular carcinoma by activating lymphocyte chemotaxis in mice. Cell Mol Gastroenterol Hepatol. 2019;7 (1):115-134. doi:10.1016/j.jcmgh.2018.08.008

18. McGuinness C, Wesley UV. Dipeptidyl peptidase IV (DPPIV), a candidate tumor suppressor gene in melanomas is silenced by promoter methylation. Front Biosci. 2008;13:2435-2443. doi:10.27 $41 / 2856$

19. Yamamoto J, Ohnuma K, Hatano R, et al. Regulation of somatostatin receptor 4-mediated cytostatic effects by CD26 in malignant pleural mesothelioma. Br J Cancer. 2014;110(9):2232-2245. doi:10.1038/ bjc. 2014.151

20. Tanaka T, Umeki K, Yamamoto I, Sakamoto F, Noguchi S, Ohtaki S. CD26 (dipeptidyl peptidase IV/DPP IV) as a novel molecular marker for differentiated thyroid carcinoma. Int $J$ Cancer. 1995;64 (5):326-331. doi:10.1002/ijc.2910640508

21. Kholova I, Ludvikova M, Ryska A, et al. Diagnostic role of markers dipeptidyl peptidase IV and thyroid peroxidase in thyroid tumors. Anticancer Res. 2003;23(2a):871-875.

22. Egawa N, Lin Y, Tabata T, et al. ABO blood type, long-standing diabetes, and the risk of pancreatic cancer. World $J$ Gastroenterol. 2013;19(16):2537-2542. doi:10.3748/wjg.v19.i16.2537

23. Ghersi G, Zhao Q, Salamone M, Yeh Y, Zucker S, Chen WT. The protease complex consisting of dipeptidyl peptidase IV and seprase plays a role in the migration and invasion of human endothelial cells in collagenous matrices. Cancer Res. 2006;66(9):4652-4661.

24. Kikkawa F, Kajiyama H, Ino K, Shibata K, Mizutani S. Increased adhesion potency of ovarian carcinoma cells to mesothelial cells by overexpression of dipeptidyl peptidase IV. Int J Cancer. 2003;105 (6):779-783. doi:10.1002/ijc.11177

25. Larrinaga G, Blanco L, Sanz B, et al. The impact of peptidase activity on clear cell renal cell carcinoma survival. Am J Physiol Renal Physiol. 2012;303(12):F1584-1591. doi:10.1152/ajprenal.00477.2 012

26. Lam CS, Cheung AH, Wong SK, et al. Prognostic significance of CD26 in patients with colorectal cancer. PLoS One. 2014;9(5): e98582. doi:10.1371/journal.pone.0098582

27. Zhang H, Lin H, Mo X, Chen G, Lin L. Synergistic relationship between dipeptidyl peptidase IV and neutral endopeptidase expression and the combined prognostic significance in osteosarcoma patients. Med Oncol. 2013;30(3):1-8. doi:10.1007/s12032-0130608-6

28. Ye C, Tian X, Yan L, Guan X, Yin S, Hao C. Elevated serum CD26 level is associated with metastasis and post-operation survival in pancreatic cancer patients. Transl Cancer Res. 2016;5(5):512-519. doi: $10.21037 /$ tcr. 2016.08 .38
OncoTargets and Therapy

\section{Publish your work in this journal}

OncoTargets and Therapy is an international, peer-reviewed, open access journal focusing on the pathological basis of all cancers, potential targets for therapy and treatment protocols employed to improve the management of cancer patients. The journal also focuses on the impact of management programs and new therapeutic

Submit your manuscript here: https://www.dovepress.com/oncotargets-and-therapy-journ agents and protocols on patient perspectives such as quality of life, adherence and satisfaction. The manuscript management system is completely online and includes a very quick and fair peer-review system, which is all easy to use. Visit http://www.dovepress.com/ testimonials.php to read real quotes from published authors. 